Article

\title{
Optimal Noise Benefit in Composite Hypothesis Testing under Different Criteria
}

\author{
Shujun Liu ${ }^{1, *}$, Ting Yang ${ }^{1}$, Mingchun Tang ${ }^{1}$, Hongqing Liu ${ }^{2}$, Kui Zhang ${ }^{1}$ \\ and Xinzheng Zhang ${ }^{1}$ \\ 1 College of Communication Engineering, Chongqing University, Chongqing 400044, China; \\ yandtt2008@foxmail.com (T.Y.); tangmingchun@cqu.edu.cn (M.T.); zk@cqu.edu.cn (K.Z.); \\ zhangxinzheng@cqu.edu.cn (X.Z.) \\ 2 Chongqing Key Lab of Mobile Communications Technology, Chongqing University of Posts and \\ Telecommunications, Chongqing 400065, China; hongqingliu@outlook.com \\ * Correspondence: liusj@cqu.edu.cn; Tel.: +86-23-6510-3544; Fax: +86-23-6510-3544 \\ Academic Editors: Julio Stern, Adriano Polpo and Kevin H. Knuth \\ Received: 30 May 2016; Accepted: 16 August 2016; Published: 19 August 2016
}

\begin{abstract}
The detectability for a noise-enhanced composite hypothesis testing problem according to different criteria is studied. In this work, the noise-enhanced detection problem is formulated as a noise-enhanced classical Neyman-Pearson (NP), Max-min, or restricted NP problem when the prior information is completely known, completely unknown, or partially known, respectively. Next, the detection performances are compared and the feasible range of the constraint on the minimum detection probability is discussed. Under certain conditions, the noise-enhanced restricted NP problem is equivalent to a noise-enhanced classical NP problem with modified prior distribution. Furthermore, the corresponding theorems and algorithms are given to search the optimal additive noise in the restricted NP framework. In addition, the relationship between the optimal noise-enhanced average detection probability and the constraint on the minimum detection probability is explored. Finally, numerical examples and simulations are provided to illustrate the theoretical results.
\end{abstract}

Keywords: additive noise; composite hypothesis testing; restricted Neyman-Pearson (NP)

\section{Introduction}

Stochastic resonance (SR) is a phenomenon where the performance of a nonlinear system can be enhanced by the presence of noise under certain circumstances. The concept of SR was first brought up by Benzi et al. in the process of exploring the periodic recurrence of ice gases [1] and since then the positive effects of noise have increasingly attracted researchers' attention in various fields, such as physics, chemistry, biology, and electronics [2-9]. The performance boost of a noise-enhanced system has also been observed in numerous signal detection problems; for example, when adjusting the background noise level or injecting additive noise to the input, the output of the system can be improved in some cases [10-15]. The improvements obtained via noise can be measured by various metrics, such as an increase in mutual information (MI) [16-19], output signal-to-noise ratio (SNR) [20-22], or detection probability [23-29], or a decrease in Bayes risk [30-32] or error probability [33].

For the hypothesis testing problem [34], the optimal additive noise to improve the performance of a suboptimal detector is usually determined according to Bayesian [24,25], Minimax [30], and Neyman-Pearson (NP) [23-26] criteria. For example, the minimization of Bayes risk obtained by adding additive noise to the observation has been investigated based on Bayesian criteria under the uniform cost assignment in [33], and it is proven that the optimal additive noise to minimize the 
error probability is a constant vector. According to the NP criteria, the maximization of detection probability obtained by adding additive noise has been considered under the constraint on the false-alarm probability. In [23], the detection of a direct current (DC) signal embedded in independent and identically distributed (i.i.d.) Gaussian mixture noise is studied, which illustrates that the detection probability of a suboptimal detector can be increased by adding an independent additive noise to the received data. In [24], a mathematical framework for the noise-enhanced binary hypothesis testing problem is formulated according to the NP criterion. The optimal noise is proven to be a randomization of no more than two discrete vectors and sufficient conditions for the increase of the detection probability via additive noise are also provided. Theorems and algorithms are also provided in [26] to search for the optimal or near-optimal SR noise that benefits the NP and inequality-constrained signal detection problems.

Composite hypothesis testing problems are often encountered in practical applications, for example, radar systems, spectrum sensing in cognitive radio networks, and non-coherent communication receivers [35,36]. In such problems, there are multiple parameters with different probabilities under each alternative hypothesis [27]. Therefore, noise that benefits composite hypothesis testing problems can also be investigated according to Bayesian, Minimax, and NP criteria. If the prior information of each parameter is completely available, the noise-enhanced Bayesian and NP approaches can be utilized. When no prior information exists, it is appropriate to select the Minimax approach to find the optimal additive noise.

Nevertheless, due to the existence of estimation error, prior information usually has some uncertainties [34]. For instance, in some cases, only part of the prior information can be utilized [37]. Accordingly, the classical Bayesian, Minimax, and NP criteria are not suitable in such cases, and a more restricted criterion should be considered in order to utilize the available partial prior information adequately. In [31], under certain constraints of the conditional risks, the optimal noise to minimize the Bayes risk is explored according to the restricted Bayesian criterion. Generally, the constraint is determined on the basis of the uncertainty in the prior information. Actually, the noise-enhanced composite hypothesis testing problem in the restricted Bayesian framework can be generalized to the Bayesian framework and Minimax framework simply by changing the value of the constraints.

In [27], the restricted NP criterion is used to find the optimal decision rule for the composite hypothesis testing problem, which focuses on maximizing the average detection probability under the constraints that the minimum detection probability cannot be less than a predefined value and the maximum false-alarm probability cannot be larger than a given level. In this way, since the constraint on the minimum detection probability is adjusted according to the uncertainty in the prior information, it not only ensures the detection probability in the worst case, but also uses the prior information effectively. Furthermore, the classical NP and Max-min criteria are special cases of the restricted NP criterion. The former aims to maximize the average detection probability based on the prior distribution with the constraint on false-alarm probability, and the latter focuses on the maximization of the minimum detection probability under the same constraint on false-alarm probability. For the case where the decision rule cannot be altered, the restricted NP criterion can also be employed to investigate the optimal additive noise for the composite hypothesis testing problem (see the study in [29]).

Inspired by the studies in $[27,29]$, the main focus of this work is to find the relations of the optimal additive noises obtained under restricted NP criterion and classical NP criterion with different prior distributions in which the noise-enhanced detection problem for composite hypothesis testing can be solved more thoroughly. In addition, the links of the average and minimum detection probabilities based on the noise-enhanced restricted NP, classical NP, and Max-min approaches are also discussed.

Our main contributions are summarized as follows. First, the noise enhanced composite hypothesis-testing problem is formulated according to the classical NP, Max-min, or restricted NP criterion when the prior information is completely known, completely unknown, or partially known, respectively. Then the detection performances obtained under different criteria are compared and the 
feasible range of the constraint on the minimum detection probability is provided. Further, under certain conditions, a special conclusion is made that the noise-enhanced restricted NP problem is equivalent to a noise-enhanced classical NP problem with a modified prior distribution and using the same constraint on the false-alarm probability. Based on this conclusion, theorems and algorithms are presented to find the optimal noise in the restricted NP framework. In addition, the relationship between the optimal noise-enhanced average detection probability and the constraint on the minimum detection probability is also explored.

Remarkably, the results in this paper can be directly applied in some specific physical environments. For instance, in order to detect a sinusoidal signal embedded in noise through exploiting the escape time of a Josephson junction [8], the escape time is regarded as the observation and the corresponding probability distribution functions (PDFs) under two hypotheses are retrieved by utilizing various nonparametric statistical techniques, such as the kernel density estimation (KDE). Generally, the distributions of the signal parameters are known with some uncertainties due to the estimation errors. In such a case, the restricted NP approach developed in this paper can be utilized. In addition, if we assume the signal parameters are completely known or unknown, the classical NP or Max-min approach can be employed, respectively, to find the corresponding noise that will enhance system performance.

The remainder of this paper is organized as follows. In Section 2, the noise-enhanced binary composite hypothesis testing problems are formulated according to the classical NP, Max-min, and restricted NP criteria, and the corresponding detection performances are compared. In Section 3, the theorems and algorithms necessary to find the modified prior distribution and the optimal additive noise are developed. In addition, some characteristics of maximum noise-enhanced average detection probability obtained in restricted NP approach are also discussed. Finally, numerical examples and simulations are presented in Section 4 to illustrate the theoretical results, and conclusions are drawn in Section 5 .

\section{Noise-Enhanced Detection}

\subsection{Problem Formulation}

Consider a classical binary composite hypothesis testing problem, given by

$$
\left\{\begin{array}{l}
H_{0}: p_{\theta}^{\mathbf{x}}(\mathbf{x}), \theta \in \Lambda_{0} \\
H_{1}: p_{\theta}^{\mathbf{x}}(\mathbf{x}), \theta \in \Lambda_{1}
\end{array}\right.
$$

where $p_{\theta}^{\mathbf{x}}(\mathbf{x})$ represents the PDF of the observation $\mathbf{x}$ for a given parameter $\theta, \mathbf{x}$ is a $N$-dimensional vector and $\mathbf{x} \in \mathbb{R}^{N}$. In addition, $\Lambda_{0}$ and $\Lambda_{1}$ are the sets of all possible values of parameter $\theta$ under $H_{0}$ and $H_{1}$, respectively, and $\Lambda_{0} \cap \Lambda_{1}=\varnothing$. The union of the two sets forms the parameter space $\Lambda$, i.e., $\Lambda=\Lambda_{0} \cup \Lambda_{1}$. The PDF for the parameter $\theta$ under $H_{i}, i=0,1$, is denoted by $\omega_{i}(\theta)$.

Studies have shown that the detection performance of a system can be improved by adding an independent additive noise $\mathbf{n}$ to the observation $\mathbf{x}$ for the case where the detector cannot be varied. The corresponding noise modified observation $\mathbf{y}$ is denoted by

$$
\mathbf{y}=\mathbf{x}+\mathbf{n}
$$

The PDF of $\mathbf{y}$ for a given parameter $\theta$ can be expressed as

$$
p_{\theta}^{\mathbf{y}}(\mathbf{x})=p_{\theta}^{\mathbf{x}}(\mathbf{x}) * p_{\mathbf{n}}(\mathbf{n})=\int_{\mathbb{R}^{N}} p_{\mathbf{n}}(\mathbf{n}) p_{\theta}^{\mathbf{x}}(\mathbf{y}-\mathbf{n}) d \mathbf{n},
$$

where $p_{\mathbf{n}}(\mathbf{n})$ denotes the PDF of the additive noise $\mathbf{n}$. Using Equation (3), the noise-modified detection and false-alarm probabilities for given parameter values are respectively defined as 


$$
\begin{aligned}
& P_{D}\left(p_{\mathbf{n}}(\mathbf{n}), \theta\right)=\int_{\mathbb{R}^{N}} \phi(\mathbf{y}) p_{\theta}^{\mathbf{y}}(\mathbf{y}) d \mathbf{y}=\int_{\mathbb{R}^{N}} p_{\mathbf{n}}(\mathbf{n}) \int_{\mathbb{R}^{N}} \phi(\mathbf{y}) p_{\theta}^{\mathbf{x}}(\mathbf{y}-\mathbf{n}) d \mathbf{y} d \mathbf{n}, \theta \in \Lambda_{1}, \\
& P_{F A}\left(p_{\mathbf{n}}(\mathbf{n}), \theta\right)=\int_{\mathbb{R}^{N}} \phi(\mathbf{y}) p_{\theta}^{\mathbf{y}}(\mathbf{y}) d \mathbf{y}=\int_{\mathbb{R}^{N}} p_{\mathbf{n}}(\mathbf{n}) \int_{\mathbb{R}^{N}} \phi(\mathbf{y}) p_{\theta}^{\mathbf{x}}(\mathbf{y}-\mathbf{n}) d \mathbf{y} d \mathbf{n}, \theta \in \Lambda_{0},
\end{aligned}
$$

where $\phi(\cdot)$ is the decision rule of the detector and also indicates the probability of choosing $H_{1}$. Due to the fact that the detector is fixed, the decision rule for the noise-modified observation $\mathbf{y}$ is the same as that for $\mathbf{x}$. For the case where the detector is fixed, one reasonable way to improve the detectability of the system is to optimize the additive noise.

\subsection{Noise-Enhanced Detection Problems under Different Criteria}

For noise-enhanced composite hypothesis testing problems, the general means is to search for the optimal additive noise to improve the system performance according to the classical NP criterion, which requires that the noise modified false-alarm probability for any possible values of parameter $\theta$ in the set $\Lambda_{0}$ should be below a certain constraint and the noise-modified average detection probability should reach the achievable maximum. In such a case, there is no link between the PDF of $\theta$ under $H_{0}$, i.e., $\omega_{0}(\theta)$, and the optimal additive noise. On the other hand, the solution of the optimal additive noise is closely related to the PDF of $\theta$ under $H_{1}$, i.e., $\omega_{1}(\theta)$.

When $\omega_{1}(\theta)$ is completely known, the optimal additive noise is usually explored according to the classical NP criterion, which is formulated by

$$
\begin{gathered}
p_{\mathbf{n}}^{c}(\mathbf{n})=\arg \max _{p_{\mathbf{n}}(\mathbf{n})} P_{D}^{a v g}\left(p_{\mathbf{n}}(\mathbf{n})\right)=\arg \max _{p_{\mathbf{n}}(\mathbf{n})} \int_{\Lambda_{1}} P_{D}\left(p_{\mathbf{n}}(\mathbf{n}), \theta\right) \boldsymbol{\omega}_{1}(\theta) d \theta \\
\text { subject to } \max _{\theta \in \Lambda_{0}} P_{F A}\left(p_{\mathbf{n}}(\mathbf{n}), \theta\right) \leq \alpha,
\end{gathered}
$$

where $p_{\mathbf{n}}^{c}(\mathbf{n})$ represents the PDF of the optimal additive noise obtained by the noise-enhanced classical NP approach, $P_{D}^{a v g}\left(p_{\mathbf{n}}(\mathbf{n})\right)=\int_{\Lambda_{1}} P_{D}\left(p_{\mathbf{n}}(\mathbf{n}), \theta\right) \omega_{1}(\theta) d \theta$ represents the noise-enhanced average detection probability based on the estimated prior probability $\omega_{1}(\theta)$, and $\alpha$ is the upper limit for the false-alarm probability.

When $\omega_{1}(\theta)$ is completely unknown, the optimal additive noise can be determined based on the Max-min criterion, where the minimum noise enhanced detection probability is maximized under the constraint on the false-alarm probability. The corresponding noise enhanced problem can be expressed by

$$
\begin{gathered}
p_{\mathbf{n}}^{m}(\mathbf{n})=\arg \max _{p_{\mathbf{n}}(\mathbf{n})} \min _{\theta \in \Lambda_{1}} P_{D}\left(p_{\mathbf{n}}(\mathbf{n}), \theta\right) \\
\text { subject to } \max _{\theta \in \Lambda_{0}} P_{F A}\left(p_{\mathbf{n}}(\mathbf{n}), \theta\right) \leq \alpha,
\end{gathered}
$$

where $p_{\mathbf{n}}^{m}(\mathbf{n})$ denotes the optimal additive noise PDF in the noise enhanced Max-min approach.

In practice, $\omega_{1}(\theta)$ is usually estimated according to previous experience and estimation errors are unavoidable, leading to some uncertainties in $\omega_{1}(\theta)$. The existence of the estimation error is often ignored when the noise enhanced detection problem based on the NP criterion is investigated. Once the Max-min criterion is applied, the previous experience cannot be utilized effectively. Therefore, it is not a suitable method for finding the optimal additive noise according to the NP criterion or the Max-min criterion directly when there are uncertainties in $\omega_{1}(\theta)$. In order to utilize previous experience and consider the uncertainty in estimation simultaneously, the restricted NP criterion proposed in [27] is utilized in this paper. Based on the estimated distribution $\omega_{1}(\theta)$, the noise-enhanced restricted NP approach seeks to maximize average detection probability by adding appropriate noise, under the constraints that the minimum detection probability stays above a certain value and the maximum false-alarm probability stays below a certain level. It should be noted that the constraint on the 
minimum detection probability can be adjusted depending on the degree of the uncertainty. As a result, the noise-enhanced problem according to the restricted NP criterion is formulated as follows:

$$
\begin{gathered}
p_{\mathbf{n}}^{\beta}(\mathbf{n})=\arg \max _{p_{\mathbf{n}}(\mathbf{n})} P_{D}^{a v g}\left(p_{\mathbf{n}}(\mathbf{n})\right) \\
\text { subject to }\left\{\begin{array}{l}
\min _{\theta \in \Lambda_{1}} P_{D}\left(p_{\mathbf{n}}(\mathbf{n}), \theta\right) \geq \beta, \\
\max _{\theta \in \Lambda_{0}} P_{F A}\left(p_{\mathbf{n}}(\mathbf{n}), \theta\right) \leq \alpha,
\end{array}\right.
\end{gathered}
$$

where $p_{\mathbf{n}}^{\beta}(\mathbf{n})$ denotes the optimal additive noise PDF in the noise-enhanced restricted NP approach, $\beta$ is the lower limit for detection probability, and an appropriate $\beta$ is chosen according to the uncertainty in $\omega_{1}(\theta)$. Generally, $\beta$ is chosen as $\beta=\varepsilon \zeta$, where $0 \leq \varepsilon \leq 1$ and $\varsigma$ represents the Max-min detection probability obtained in the noise-enhanced Max-min approach, i.e., $\varsigma=\min _{\theta \in \Lambda_{1}} P_{D}\left(p_{\mathbf{n}}^{m}(\mathbf{n}), \theta\right)=$ $\max _{p_{\mathbf{n}}(\mathbf{n})} \min _{\theta \in \Lambda_{1}} P_{D}\left(p_{\mathbf{n}}(\mathbf{n}), \theta\right)$.

When the prior information about $\omega_{1}(\theta)$ is completely unknown, we set $\varepsilon=1$ and the noise-enhanced detection problem in the restricted NP framework is reduced to that in the Max-min framework. When $\omega_{1}(\theta)$ is completely known, we set $\varepsilon=0$ and the noise-enhanced detection problem in the restricted NP framework becomes the noise-enhanced problem in the classical NP framework. As a result, the noise-enhanced Max-min problem and classical NP problem can be regarded as two special cases of the noise-enhanced restricted NP problem.

\subsection{Analysis on the Noise-Enhanced Detectability}

In this subsection, the relationships among the different noise-enhanced approaches are clarified in detail. We first compare the detection performances obtained via additive noise under different criteria and then determine the feasible range of $\beta$, which is the lower limit of the detection probability for any $\theta \in \Lambda_{1}$.

In the noise-enhanced restricted NP framework, the maximization of the average detection probability needs to consider the constraints on the minimum detection probability for $\theta \in \Lambda_{1}$ and the maximum false-alarm probability for $\theta \in \Lambda_{0}$ simultaneously. On the other hand, the aim of the noise-enhanced classical NP problem is to find the additive noise that maximizes the average detection probability with only the constraint on false-alarm probability, while ignoring any constraints on the minimum detection probability. Generally speaking, the maximum average detection probability obtained by the noise-enhanced restricted NP approach is less than or equal to that obtained by the noise-enhanced classical NP approach, and the minimum detection probability obtained by the noise-enhanced restricted NP approach is greater than or equal to that obtained by the noise-enhanced classical NP approach. For the noise enhanced Max-min approach, however, the aim is to search the additive noise that achieves the maximum of the minimum detection probability under the constraint on false-alarm probability. Therefore, the minimum detection probability obtained by the noise-enhanced Max-min approach is greater than or equal to that obtained by the noise enhanced restricted NP approach.

Based on the discussions above, in what follows, the detectability in the noise-enhanced restricted NP framework is analyzed when $\beta$ takes different values. From the definitions given in Section 2.2, $p_{\mathbf{n}}^{\beta}(\mathbf{n}), p_{\mathbf{n}}^{c}(\mathbf{n})$, and $p_{\mathbf{n}}^{m}(\mathbf{n})$ are the optimal additive noise PDFs corresponding to the noise-enhanced restricted NP, classical NP, and Max-min approaches, respectively. In order to facilitate the analysis that follows, we define

$$
\begin{gathered}
C=\min _{\theta \in \Lambda_{1}} P_{D}\left(p_{\mathbf{n}}^{c}(\mathbf{n}), \theta\right) \\
M=\min _{\theta \in \Lambda_{1}} P_{D}\left(p_{\mathbf{n}}^{m}(\mathbf{n}), \theta\right)
\end{gathered}
$$


i.e., $C$ and $M$ are the minimum detection probabilities corresponding to the noise-enhanced classical NP approach and Max-min approach for $\theta \in \Lambda_{1}$, respectively.

If $\beta<C$, the noise-enhanced restricted NP problem is simplified to the noise enhanced classical NP problem. It is obvious that the constraint enforced by $\beta$ is ineffective in this case. Accordingly, the optimal additive noise and the maximum noise-modified average detection probability obtained by the restricted NP approach are the same as those obtained by the classical NP approach, i.e., $p_{\mathbf{n}}^{\beta}(\mathbf{n})=p_{\mathbf{n}}^{c}(\mathbf{n})$ and $P_{D}^{a v g}\left(p_{\mathbf{n}}^{\beta}(\mathbf{n})\right)=P_{D}^{a v g}\left(p_{\mathbf{n}}^{c}(\mathbf{n})\right)$. Furthermore, based on the formulation of the noise enhanced Max-min approach, $M$ is the achievable maximum of the minimum detection probability for any $\theta \in \Lambda_{1}$ obtained by adding additive noise under the constraint on false-alarm probability. Therefore, there are no additive noises that satisfy the condition of $\min _{\theta \in \Lambda_{1}} P_{D}\left(p_{\mathbf{n}}(\mathbf{n}), \theta\right)>M$. In other words, it is not suitable to set $\beta>M$. As a result, the value of $\beta$ should be selected in the interval $[C, M]$, otherwise the constraint on the minimum detection probability will be meaningless.

\section{Optimal Additive Noises in Restricted NP and Classical NP Frameworks}

In this section, we shall further explore the connections implied in the optimal additive noises obtained by the restricted NP and classical NP approaches under certain conditions. The related characteristics are discussed in order to develop an algorithm that determines the optimal additive noise under the restricted NP framework. For the convenience of discussions, we define

$$
\begin{gathered}
f_{\theta}(\mathbf{n})=\int_{\mathbb{R}^{N}} \phi(\mathbf{y}) p_{\theta}^{\mathbf{x}}(\mathbf{y}-\mathbf{n}) d \mathbf{y} \\
f_{1}(\mathbf{n})=\int_{\Lambda_{1}} f_{\theta}(\mathbf{n}) \omega_{1}(\theta) d \theta .
\end{gathered}
$$

With these notations, the optimization problem in (10) and (11) can be reformulated as

$$
\begin{gathered}
\max _{p_{\mathbf{n}}(\mathbf{n})} \int_{\Lambda_{1}} P_{D}(\mathbf{n}, \theta) \omega_{1}(\theta) d \theta=\max _{p_{\mathbf{n}}(\mathbf{n})} \int_{\mathbb{R}^{N}} p_{\mathbf{n}}(\mathbf{n}) f_{1}(\mathbf{n}) d \mathbf{n} \\
\text { subject to }\left\{\begin{array}{l}
\min _{\theta \in \Lambda_{1}} \int_{\mathbb{R}^{N}} p_{\mathbf{n}}(\mathbf{n}) f_{\theta}(\mathbf{n}) d \mathbf{n} \geq \beta \\
\max _{\theta \in \Lambda_{0}} \int_{\mathbb{R}^{N}} p_{\mathbf{n}}(\mathbf{n}) f_{\theta}(\mathbf{n}) d \mathbf{n} \leq \alpha
\end{array}\right.
\end{gathered}
$$

Actually, the problem in Equations (16) and (17) can be expressed in alternative form as follows:

$$
\begin{gathered}
\max _{p_{\mathbf{n}}(\mathbf{n})}\left\{\eta \int_{\mathbb{R}^{N}} p_{\mathbf{n}}(\mathbf{n}) f_{1}(\mathbf{n}) d \mathbf{n}+(1-\eta) \min _{\theta \in \Lambda_{1}} \int_{\mathbb{R}^{N}} p_{\mathbf{n}}(\mathbf{n}) f_{\theta}(\mathbf{n}) d \mathbf{n}\right\} \\
\max _{\theta \in \Lambda_{0}} \int_{\mathbb{R}^{N}} p_{\mathbf{n}}(\mathbf{n}) f_{\theta}(\mathbf{n}) d \mathbf{n} \leq \alpha,
\end{gathered}
$$

where $\eta$ is a parameter selected depending on $\beta$ and $0 \leq \eta \leq 1$.

\subsection{Characteristics of the Optimal Additive Noise}

According to Equations (18) and (19), Theorem 1 shows some characteristics of the optimal additive noise obtained by the restricted NP approach.

Theorem 1. Define a PDF of $\theta$ under $H_{1}$ as $r_{1}(\theta)=\eta \omega_{1}(\theta)+(1-\eta) g(\theta)$, where $g(\theta)$ is any valid PDF. If there exists a PDF $p_{\mathbf{n}}^{*}(\mathbf{n})$ such that

$$
\int_{\mathbb{R}^{N}} p_{\mathbf{n}}^{*}(\mathbf{n}) \int_{\Lambda_{1}} f_{\theta}(\mathbf{n}) g(\theta) d \theta d \mathbf{n}=\min _{\theta \in \Lambda_{1}} \int_{\mathbb{R}^{N}} p_{\mathbf{n}}^{*}(\mathbf{n}) f_{\theta}(\mathbf{n}) d \mathbf{n},
$$


where $p_{\mathbf{n}}^{*}(\mathbf{n})$ is the optimal additive noise PDF that maximizes the average detection probability based on $r_{1}(\theta)$ under the constraint that $\max _{\theta \in \Lambda_{1}} \int_{\mathbb{R}^{N}} p_{\mathbf{n}}(\mathbf{n}) f_{\theta}(\mathbf{n}) d \mathbf{n} \leq \alpha$, then $p_{\mathbf{n}}^{*}(\mathbf{n})$ is the optimal solution of Equations (18) and (19). The proof is presented in Appendix A.

Theorem 1 implies that the solution of the noise-enhanced restricted NP problem is the same as that of a noise-enhanced classical NP problem under certain conditions. Specifically, if there exists a PDF $g(\theta)$ that satisfies the condition in Equation (20), the noise-enhanced restricted NP problem shown in (18) and (19) is equivalent to the noise-enhanced classical NP problem based on the probability distribution of $\theta$ under $H_{1}$, denoted by $r_{1}(\theta)=\eta \omega_{1}(\theta)+(1-\eta) g(\theta)$. As discussed above, the definition of $p_{\mathbf{n}}^{*}(\mathbf{n})$ can be formulated as

$$
\begin{gathered}
p_{\mathbf{n}}^{*}(\mathbf{n})=\arg \max _{p_{\mathbf{n}}(\mathbf{n})} \int_{\Lambda_{1}} P_{D}\left(p_{\mathbf{n}}(\mathbf{n}), \theta\right) r_{1}(\theta) d \theta \\
\text { subject to } \max _{\theta \in \Lambda_{1}} \int_{\mathbb{R}^{N}} p_{\mathbf{n}}(\mathbf{n}) f_{\theta}(\mathbf{n}) d \mathbf{n} \leq \alpha .
\end{gathered}
$$

The form of the optimal additive noise PDF $p_{\mathbf{n}}^{*}(\mathbf{n})$ is proven as a randomization of no more than $M+1$ discrete vectors and the corresponding algorithm has been provided in [28], where $M$ is the number of $\theta$ in the set $\Lambda_{0}$.

The following corollary shows the link between the optimization noise-enhanced problem in Equations (18) and (19) and that in (10) and (11).

Corollary 1. Under the conditions in Theorem 1, if $\min _{\theta \in \Lambda_{1}} \int_{\mathbb{R}^{N}} p_{\mathbf{n}}^{*}(\mathbf{n}) f_{\theta}(\mathbf{n}) d \mathbf{n}=\beta, p_{\mathbf{n}}^{*}(\mathbf{n})$ is the optimal additive noise PDF corresponding to the optimization problem described in Equations (10) and (11). The proof is omitted here and provided in Appendix A.

First, Corollary 1 illustrates that $p_{\mathbf{n}}^{*}(\mathbf{n})$ is the solution of the optimization problem described in Equations (16) and (17), or the one in (10) and (11), when the additive noise PDF $p_{\mathbf{n}}^{*}(\mathbf{n})$ in Theorem 1 satisfies $\min _{\theta \in \Lambda_{1}} \int_{\mathbb{R}^{N}} p_{\mathbf{n}}^{*}(\mathbf{n}) f_{\theta}(\mathbf{n}) d \mathbf{n}=\beta$. In such a case, when the noise-modified average detection probability based on $\omega_{1}(\theta)$ reaches the achievable maximum, the corresponding minimum detection probability for all $\theta \in \Lambda_{1}$ is equal to $\beta$. Second, for any $\eta$, the corresponding $\beta$ can be calculated based on the equality in Corollary 1.

In addition, in order to find $r_{1}(\theta)$, we first define a family of $r_{1}(\theta)$ 's PDF of the following form:

$$
\hat{r}_{1}(\theta)=\hat{\eta} \omega_{1}(\theta)+(1-\hat{\eta}) \hat{g}(\theta),
$$

where $\hat{\eta}$ and $1-\hat{\eta}$ represent the weights of $\omega_{1}(\theta)$ and $\hat{g}(\theta)$ in $\hat{r}_{1}(\theta)$, respectively, $0 \leq \hat{\eta} \leq 1$, and $\hat{g}(\theta)$ is any valid PDF. Theorem 2 below states a conclusion that $r_{1}(\theta)$ in Theorem 1 is the PDF corresponding to the minimum noise-enhanced average detection probability, which is among the family of PDFs with the form of $\hat{r}_{1}(\theta)$.

Theorem 2. Under the conditions in Theorem $1, r_{1}(\theta)=\eta \omega_{1}(\theta)+(1-\eta) g(\theta)$ is the PDF that minimizes the noise-modified average detection probability among all probability distributions of the form $\hat{r}_{1}(\theta)=\hat{\eta} \omega_{1}(\theta)+$ $(1-\hat{\eta}) \hat{g}(\theta)$, where $\hat{\eta} \geq \eta, \theta \in \Lambda_{1}$, and $\hat{g}(\theta)$ is any PDF. In other words, the following inequality holds:

$$
\int_{\mathbb{R}^{N}} p_{\mathbf{n}}^{*}(\mathbf{n}) \int_{\Lambda_{1}} f_{\theta}(\mathbf{n}) r_{1}(\theta) d \theta d \mathbf{n} \leq \int_{\mathbb{R}^{N}} \hat{p}_{\mathbf{n}}^{*}(\mathbf{n}) \int_{\Lambda_{1}} f_{\theta}(\mathbf{n}) \hat{r}_{1}(\theta) d \theta d \mathbf{n},
$$

where $p_{\mathbf{n}}^{*}(\mathbf{n})$ and $\hat{p}_{\mathbf{n}}^{*}(\mathbf{n})$ are the optimal additive noise PDFs obtained by the classical NP approach corresponding to $r_{1}(\theta)$ and $\hat{r}_{1}(\theta)$, respectively. The proof is omitted here and provided in Appendix A. 
Obviously, $r_{1}(\theta)$ is a special case of $\hat{r}_{1}(\theta)$, and therefore we can search the explicit expression of $r_{1}(\theta)$ and the optimal additive noise under the restricted NP criterion by exploiting the conclusion in Theorem 2. In addition, since $\eta$ is a special value of $\hat{\eta}$, for practical applications we only need to consider the case of $\hat{\eta}=\eta$. The detailed algorithm is presented in the next subsection.

\subsection{Algorithm for the Optimal Additive Noise}

The analysis in Subsection 3.1 indicates that, in order to solve the noise-enhanced restricted NP problem in Equations (10) and (11), we need to obtain a distribution $g(\theta)$ and the optimal additive noise PDF $p_{\mathbf{n}}^{*}(\mathbf{n})$ corresponding to $r_{1}(\theta)$ in the classical NP framework to satisfy the conditions in Theorem 1 . To achieve this aim, the condition of (20) in Theorem 1 is rewritten as

$$
\int_{\Lambda_{1}} g(\theta) P_{D}\left(p_{\mathbf{n}}^{*}(\mathbf{n}), \theta\right) d \theta=\min _{\theta \in \Lambda_{1}} P_{D}\left(p_{\mathbf{n}}^{*}(\mathbf{n}), \theta\right) .
$$

Equation (25) reveals that $g(\theta)$ only assigns nonzero values where $\theta$ corresponds to the global minimum of $P_{D}\left(p_{\mathbf{n}}^{*}(\mathbf{n}), \theta\right)$. It is assumed that the value of $\theta$ that achieves the global minimum of $P_{D}\left(p_{\mathbf{n}}^{*}(\mathbf{n}), \theta\right)$ is unique and thus $g(\theta)$ can be expressed as

$$
g(\theta)=\delta\left(\theta-\theta_{1}\right)
$$

where $\theta_{1}$ represents the unique $\theta$ that minimizes $P_{D}\left(p_{\mathbf{n}}^{*}(\mathbf{n}), \theta\right)$. Based on this assumption, the following algorithm is provided to find $p_{\mathbf{n}}^{*}(\mathbf{n})$ and $\theta_{1}$.

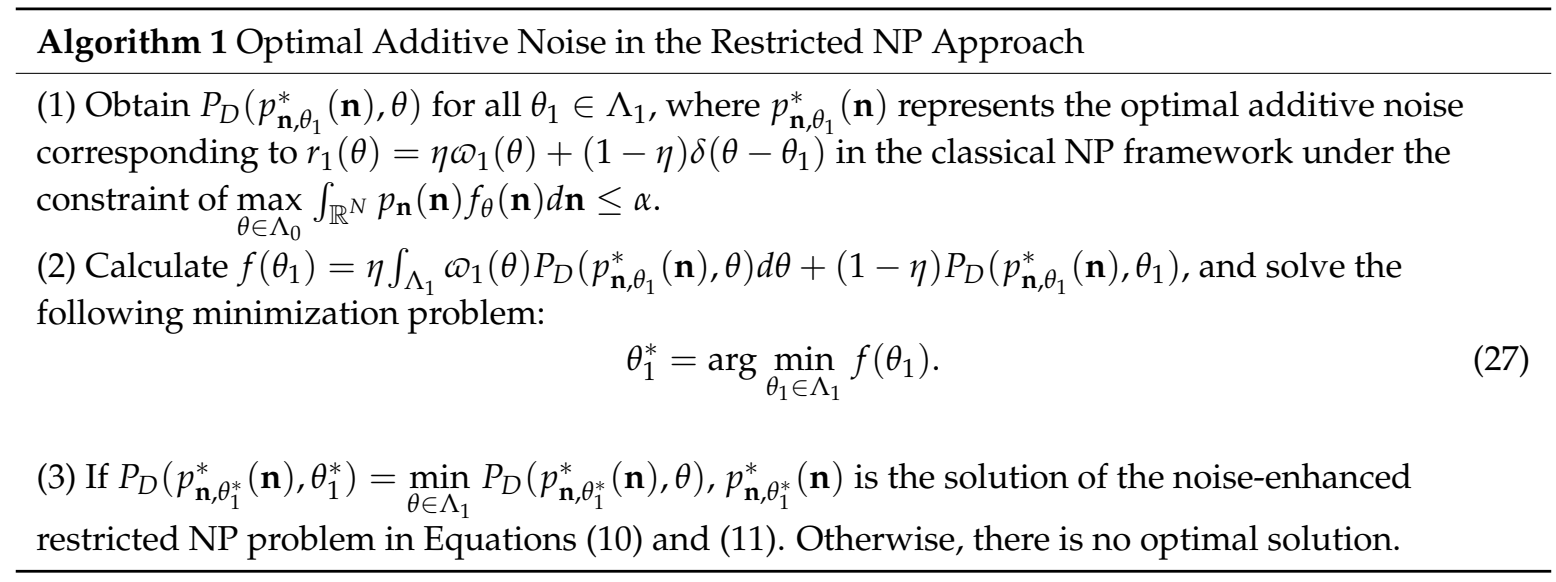

In Algorithm $1, f\left(\theta_{1}\right)$ can be regarded as the average detection probability corresponding to $r_{1}(\theta)=\eta \omega_{1}(\theta)+(1-\eta) \delta\left(\theta-\theta_{1}\right)$ and Step (2) is satisfied based on Theorem 2. It should be noted that the $\theta_{1}^{*}$ obtained in Equation (27) may be not unique. When that happens, for any $\theta_{1}^{*}$ and if the corresponding optimal additive noise $p_{\mathbf{n}, \theta_{1}^{*}}^{*}(\mathbf{n})$ satisfies the equality in Step (3), $p_{\mathbf{n}, \theta_{1}^{*}}^{*}(\mathbf{n})$ is the solution of the noise-enhanced restricted NP problem, according to Theorem 1.

If there is more than one value of $\theta$ corresponding to the global minimum $P_{D}\left(p_{\mathbf{n}}^{*}(\mathbf{n}), \theta\right)$, the expression of $g(\theta)$ can be written as

$$
g(\theta)=\sum_{i=1}^{L} \mu_{i} \delta\left(\theta-\theta_{i}\right)
$$

where $\mu_{i} \geq 0, \sum_{i=1}^{L} \mu_{i}=1, \theta_{i}$ and $L$ are the value and the number of $\theta$ corresponding to the global minimum $P_{D}\left(p_{\mathbf{n}}^{*}(\mathbf{n}), \theta\right)$, respectively. In such a case, let $v$ represent the vector consisting of all the 
unknown parameters in $g(\theta)$, i.e., $v=\left[\left(\mu_{1}, \theta_{1}\right), \cdots,\left(\mu_{L}, \theta_{L}\right)\right]$. Accordingly, Step (2) in the algorithm can be updated by

$$
v^{*}=\arg \min _{v} f(v)=\arg \min _{v}\left\{\eta \int_{\Lambda_{1}} \omega_{1}(\theta) P_{D}\left(p_{\mathbf{n}, v}^{*}(\mathbf{n}), \theta\right) d \theta+(1-\eta) \sum_{i=1}^{L} \mu_{i} P_{D}\left(p_{\mathbf{n}, v}^{*}(\mathbf{n}), \theta_{i}\right)\right\},
$$

where $p_{\mathbf{n}, v}^{*}(\mathbf{n})$ denotes the optimal additive noise PDF corresponding to $r_{1}(\theta)=\eta \omega_{1}(\theta)+$ $(1-\eta) \sum_{i=1}^{L} \mu_{i} \delta\left(\theta-\theta_{i}\right)$ obtained by the noise-enhanced classical NP approach. Moreover, if the condition of $P_{D}\left(p_{\mathbf{n}, v^{*}}^{*}(\mathbf{n}), v^{*}\right)=\min _{\theta \in \Lambda_{1}} P_{D}\left(p_{\mathbf{n}, v^{*}}^{*}(\mathbf{n}), v\right)$ in Step (3) holds, the corresponding $p_{\mathbf{n}, v^{*}}^{*}(\mathbf{n})$ is indeed the solution of Equations (10) and (11). From the analysis above, compared to the case where the global minimum of $P_{D}\left(p_{\mathbf{n}}^{*}(\mathbf{n}), \theta\right)$ is achieved by a unique $\theta$, the computational complexity is increased significantly. In order to overcome this problem, some global optimization algorithms, for example the ant colony algorithm (ACO), genetic algorithm (GA), and particle swarm optimization algorithm (PSO), can be utilized to find $v^{*}$.

If there are infinite values of $\theta$ that achieve the global minimum $P_{D}\left(p_{\mathbf{n}}^{*}(\mathbf{n}), \theta\right)$, the Parzen window density estimation can be used to approximate the form of $g(\theta)$ that solves the noise-enhanced restricted NP problem. Specifically, $g(\theta)$ can be approximately denoted by a convex combination of multiple window functions, given by

$$
g(\theta) \approx \sum_{i=1}^{W} \xi_{i} \varphi\left(\theta-\theta_{i}\right)
$$

The noise-enhanced restricted NP problem can now be solved by updating the algorithm through replacing $v=\left[\left(\mu_{1}, \theta_{1}\right), \cdots,\left(\mu_{L}, \theta_{L}\right)\right]$ with $v=\left[\left(\xi_{1}, \theta_{1}\right), \cdots,\left(\xi_{W}, \theta_{W}\right)\right]$ and redefining $p_{\mathbf{n}, v}^{*}(\mathbf{n})$ as the optimal additive noise corresponding to $r_{1}(\theta)=\eta \omega_{1}(\theta)+(1-\eta) \sum_{i=1}^{W} \xi_{i} \varphi\left(\theta-\theta_{i}\right)$ obtained by the noise-enhanced classical NP approach.

In practical applications, the value and number of $\theta$ to maximize $P_{D}\left(p_{\mathbf{n}}^{*}(\mathbf{n}), \theta\right)$ are generally unknown in advance. Therefore, we usually first assume that there exists only one $\theta$ corresponding to the global minimum $P_{D}\left(p_{\mathbf{n}}^{*}(\mathbf{n}), \theta\right)$, and then $\theta_{1}^{*}$ can be solved according to the Algorithm 1 . If the result matches the condition in Step (3), the noise-enhanced restricted NP problem in Equations (10) and (11) has been solved. Otherwise, according to the algorithm and in order to solve the global minimum, the number of values of $\theta$ will be incrementally increased until the optimal noise that satisfies the noise-enhanced restricted NP criterion is obtained.

\subsection{Noise-Enhanced Average Detection Probability on $\beta$}

As concluded in Section 2.2, $\beta$ is ineffective for $\beta<C$ and meaningless for $\beta>M$. Therefore, in the rest of this section we shall only consider the noise-enhanced average detection probability $P_{D}^{a v g}\left(p_{\mathbf{n}}^{\beta}(\mathbf{n})\right)$ obtained under the restricted NP framework for $\beta \in[C, M]$. Prior to discussing the relation between $P_{D}^{a v g}\left(p_{\mathbf{n}}^{\beta}(\mathbf{n})\right)$ and $\beta$ for $\beta \in[C, M]$, an important concept should be explained explicitly first, namely, that for any $C<\beta<M$ we have

$$
\min _{\theta \in \Lambda_{1}} P_{D}\left(p_{\mathbf{n}}^{\beta}(\mathbf{n}), \theta\right)=\beta
$$

We now employ the contradiction method to illustrate the conclusion in Equation (31). It is assumed that $\min _{\theta \in \Lambda_{1}} P_{D}\left(p_{\mathbf{n}}^{\beta}(\mathbf{n}), \theta\right)>\beta$. In this case, there exists an additive noise $\operatorname{PDF} p_{\mathbf{n}}(\mathbf{n})=\lambda p_{\mathbf{n}}^{c}(\mathbf{n})+$ $(1-\lambda) p_{\mathbf{n}}^{\beta}(\mathbf{n}), 0<\lambda<1$, that satisfies $\min _{\theta \in \Lambda_{1}} P_{D}\left(p_{\mathbf{n}}(\mathbf{n}), \theta\right)=\beta$, where $p_{\mathbf{n}}^{c}(\mathbf{n})$ is the optimal additive noise PDF in the noise-enhanced classical NP approach. According to the definition of $p_{\mathbf{n}}^{c}(\mathbf{n}), \min _{\theta \in \Lambda_{1}} P_{D}\left(p_{\mathbf{n}}^{c}(\mathbf{n}), \theta\right)=C<\beta$ and $P_{D}^{a v g}\left(p_{\mathbf{n}}^{c}(\mathbf{n})\right)>P_{D}^{a v g}\left(p_{\mathbf{n}}^{\beta}(\mathbf{n})\right)$. Consequently, $P_{D}^{a v g}\left(p_{\mathbf{n}}(\mathbf{n})\right)>$ 
$P_{D}^{a v g}\left(p_{\mathbf{n}}^{\beta}(\mathbf{n})\right)$, which contradicts the definition of $p_{\mathbf{n}}^{\beta}(\mathbf{n})$. Therefore, the minimum of $P_{D}\left(p_{\mathbf{n}}^{\beta}(\mathbf{n}), \theta\right)$ for $\theta \in \Lambda_{1}$ cannot be greater than $\beta$, i.e., $\min _{\theta \in \Lambda_{1}} P_{D}\left(p_{\mathbf{n}}^{\beta}(\mathbf{n}), \theta\right)=\beta$. Theorem 3 now shows the link between $P_{D}^{a v g}\left(p_{\mathbf{n}}^{\beta}(\mathbf{n})\right)$ and $\beta, \beta \in[C, M]$.

Theorem 3. When $\beta \in[C, M]$, the maximum noise-modified detection probability $P_{D}^{a v g}\left(p_{\mathbf{n}}^{\beta}(\mathbf{n})\right)$ obtained by the restricted NP approach is a strictly decreasing and concave function of $\beta$. The proof is presented in Appendix A.

Theorem 3 illustrates that the maximum noise-enhanced average detection probability increases monotonically as $\beta$ decreases towards $C$. In other words, the average detection probability can be improved by reducing the constraint on the minimum detection probability in practical applications. On the other hand, $\beta$ is chosen according to the estimation uncertainty. When the uncertainty decreases, we can select a smaller $\beta$. Therefore, Theorem 3 also indicates that the higher the validity of estimation, the greater the average detection probability that could be obtained by adding additive noise.

\section{Numerical Examples and Simulation Analysis}

In this section, the conclusions investigated in the previous sections will be verified through a practical example and the corresponding simulations. The two hypotheses are considered here and given by

$$
\left\{\begin{array}{l}
H_{0}: x=v \\
H_{1}: x=\Theta+v
\end{array}\right.
$$

where $x \in R, \Theta$ is a parameter with some uncertainties, and $v$ is a symmetric Gaussian mixture noise with the PDF of form

$$
p_{v}(v)=\sum_{i=1}^{K} \omega_{i} \gamma\left(v ; \mu_{i}, \sigma_{i}^{2}\right),
$$

where $\omega_{i}>0, \sum_{i=1}^{K} \omega_{i}=1$, and $\gamma\left(v ; \mu_{i}, \sigma_{i}^{2}\right)=\left(1 / \sqrt{2 \pi \sigma_{i}^{2}}\right) \exp \left[-\left(v-\mu_{i}\right)^{2} / 2 \sigma_{i}{ }^{2}\right]$. In this example, the parameter $\Theta$ is modeled as a random variable with the PDF

$$
\omega_{1}(\theta)=\tau \delta(\theta-A)+(1-\tau) \delta(\theta+A),
$$

where $A$ is a known positive constant, and $\tau$ is known, but with some uncertainties. The sets of $\Theta$ under $H_{0}$ and $H_{1}$ are $\Lambda_{0}=\{0\}$ and $\Lambda_{1}=\{A,-A\}$, respectively. The conditional PDF of $x$ for any given value of $\Theta=\theta$ can be calculated by

$$
p_{\theta}(x)=\sum_{i=1}^{K} \omega_{i}\left(1 / \sqrt{2 \pi \sigma_{i}^{2}}\right) \exp \left[-\left(x-\theta-\mu_{i}\right)^{2} / 2 \sigma_{i}^{2}\right] .
$$

The decision rule of the detector is given as

$$
\phi(y)=\left\{\begin{array}{ll}
0, & -A / 2<y<A / 2 \\
1, & y \leq-A / 2, y>A / 2
\end{array},\right.
$$

where $y=x+n$ and $n$ denotes the independent additive noise. Correspondingly,

$$
\begin{gathered}
f_{\theta}(n)=\int_{\mathbb{R}^{N}} \phi(y) p_{\theta}(y-n) d y=\sum_{i=1}^{K} \omega_{i}\left[Q\left(\frac{A / 2+n+\theta+\mu_{i}}{\sigma_{i}}\right)+Q\left(\frac{A / 2-n-\theta-\mu_{i}}{\sigma_{i}}\right)\right] \\
f_{1}(n)=\tau f_{A}(n)+(1-\tau) f_{-A}(n),
\end{gathered}
$$

where $Q(x)=\int_{x}^{+\infty}(1 / \sqrt{2 \pi}) \exp \left(-t^{2} / 2\right) d t$. 
In this example, let $K=4$ and suppose that the means of the symmetric Gaussian components in the mixture noise are [ $\left.\begin{array}{llll}0.1 & 0.6 & -0.6-0.1\end{array}\right]$, with corresponding weights of [ $\left.\begin{array}{llll}0.35 & 0.15 & 0.15 & 0.35\end{array}\right]$. In addition, it is assumed that the variances of the symmetric Gaussian components in the mixture noise are the same, namely $\sigma_{i}=\sigma$ for $i=1, \ldots, 4$. From the analysis in Section 3.2, we assume $g(\theta)=\delta\left(\theta-\theta_{1}\right)$ first, and then the algorithm presented in Equation (28) can be utilized to find $\theta_{1}^{*}$ and the corresponding optimal additive noise. If the solution satisfies the condition in Step (3), the optimal additive noise is obtained by the restricted NP approach. Otherwise, it should be assumed that $g(\theta)=\lambda \delta(\theta-A)+(1-\lambda) \delta(\theta+A)$, where $0<\lambda<1$. In this case, we only need to utilize the algorithm to determine the unknown parameter $\lambda$, which minimizes the average detection probability based on the prior distribution $r_{1}(\theta)=\eta \omega_{1}(\theta)+(1-\eta) g(\theta)$, and the optimal additive noise can be obtained accordingly.

Figure 1 compares the maximum noise-modified average detection probabilities obtained by the restricted NP approach versus $\beta$ for $\tau=0.9,0.8$, and 0.7 when $A=1, \sigma=0.2$, and $\alpha=0.3$. Due to the symmetry of the background noise, the original average detection probability is independent of $\tau$ and equals 0.8878. Compared with the data in Figure 1, the average detection probability can be improved by adding additive noise under the constraints that the false-alarm probability should not be greater than $\alpha$ and the minimum detection probability should not be less than $\beta$. As defined in Equation (12), the three values of $\beta$, i.e., $C_{1}, C_{2}$, and $C_{3}$, in Figure 1 are the minimum detection probabilities obtained by the noise-enhanced classical NP approach for $\tau=0.9,0.8$ and 0.7 , respectively. In this example, the value of $C$ increases with the decrease of $\tau$, i.e., $C_{1}<C_{2}<C_{3}$. From Figure $1, M$ is the minimum detection probability obtained by the noise enhanced Max-min approach. At the same time, $M$ is the achievable maximum of the minimum detection probability in the three different noise-enhanced approaches, which is consistent with the definition given in Equation (13). Additionally, the value of $M$ is independent of $\tau$ and $M=0.8988$.

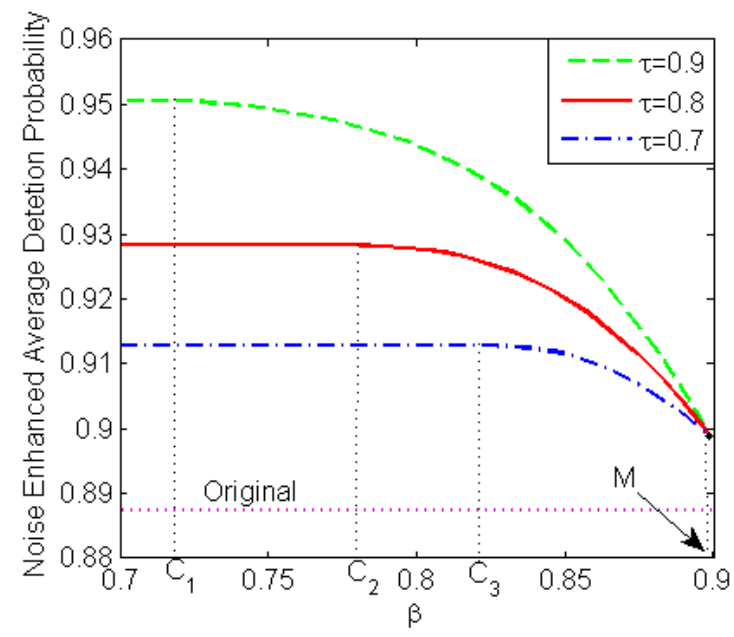

Figure 1. Noise-enhanced average detection probabilities versus $\beta$ obtained by the restricted NP approach for $\tau=0.9,0.8$ and 0.7 when $A=1, \sigma=0.2$, and $\alpha=0.3$.

With the decrease of $\tau$, the maximum noise-modified average detection probability obtained by the restricted NP approach decreases, and the corresponding value of $C$ increases and approaches $M$. When $C=M$, both the noise-enhanced restricted NP problem and the noise-enhanced classical NP problem are equivalent to the noise-enhanced Max-min problem. If $\beta \in[C, M]$ for a given $\tau$, the maximum noise-modified average detection probability obtained by the restricted NP approach decreases with the increase of $\beta$. If $\beta<C$, the optimal solution for the noise-enhanced restricted NP problem is the same as that for the noise-enhanced classical NP problem. Therefore, the maximum 
noise-modified average detection probability obtained by the restricted NP approach remains constant for $\beta<C$ and is equal to that obtained by the noise-enhanced classical NP approach.

Figure 2 depicts the maximum noise-enhanced average detection probabilities and the corresponding minimum detection probabilities versus $\eta$ obtained by the restricted NP approach for $\tau=0.9,0.8$, and 0.7 when $A=1, \sigma=0.2$, and $\alpha=0.3$. As shown in Figure 2, for a given value of $\tau$, when the value of $\eta$ exceeds a certain threshold $\eta_{0}$, the maximum noise-enhanced average detection probability increases and the corresponding minimum detection probability decreases with an increase of $\eta$. According to the analysis in the previous sections, the value of $\eta_{0}$ is specified by $\beta=M$. When $\eta<\eta_{0}$, the noise-enhanced restricted NP problem is equivalent to the noise-enhanced Max-min problem, and the corresponding maximum noise-enhanced average detection probability is the same as the minimum detection probability, and equal to $M$. In this case, the value of $M$ is independent of $\tau$, which also agrees with the conclusion in Figure 1. In addition, the value of $\eta_{0}$ decreases as $\tau$ increases, shown in Figure 2, namely $\eta_{1}<\eta_{2}<\eta_{3}$, where $\eta_{1}, \eta_{2}$, and $\eta_{3}$ are the values of $\eta_{0}$ corresponding to $\tau=0.9,0.8$, and 0.7 , respectively. In other words, a larger $\tau$ provides a bigger feasible range of $\eta$. In general, the maximum noise-enhanced average detection probability obtained by the restricted NP approach increases, while the corresponding minimum detection probability decreases with an increase of $\tau$ for any $\eta>\eta_{0}$.

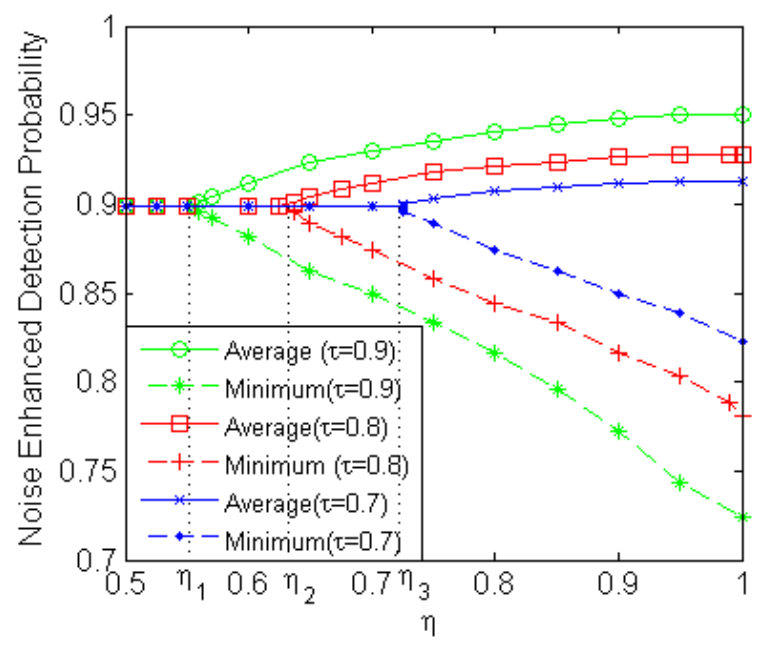

Figure 2. Noise-enhanced average and minimum detection probabilities versus $\eta$ obtained by the restricted NP approach for $\tau=0.9,0.8$, and 0.7 when $A=1, \sigma=0.2$, and $\alpha=0.3$.

Figure 3 illustrates the average detection probabilities for $\alpha=0.25$ and 0.35 obtained by the noise-enhanced restricted NP approach and the original detector versus $\sigma$ when $A=1, \tau=0.7$ and $\beta=0.8$. It is seen that with the increase of $\sigma$ the original average detection probability initially remains constant, then decreases gradually after reaching the maximum. On the other hand, the average detection probability obtained by the noise-enhanced restricted NP approach initially remains constant then gradually decreases. In addition, the smaller the value of $\sigma$, the greater the gain obtained by adding noise. Compared with the average detection probabilities obtained by the noise-enhanced restricted NP approach under the constraints that $\alpha=0.35$ and 0.25 , respectively, the noise-enhanced average detection probability increases as $\alpha$ increases, which again agrees with the theoretical analysis.

Figure 4 compares the maximum noise-enhanced average detection probabilities and the corresponding minimum noise modified detection probabilities, which are obtained by the restricted NP approach for $\eta=0.8$ and 0.7, classical NP, Max-min approaches, and the original detector, versus $\sigma$ when $A=1, \tau=0.8$, and $\alpha=0.25$. It should be noted that the average detection probability is equal to the minimum detection probability for the noise-enhanced Max-min approach and the original detector. As shown in Figure 4, the noise-enhanced classical NP approach produces the highest 
average detection probability and the lowest minimum detection probability, while the noise-enhanced Max-min approach obtains the maximum of the minimum detection probability. The results are consistent with the analysis in Section 3.3.

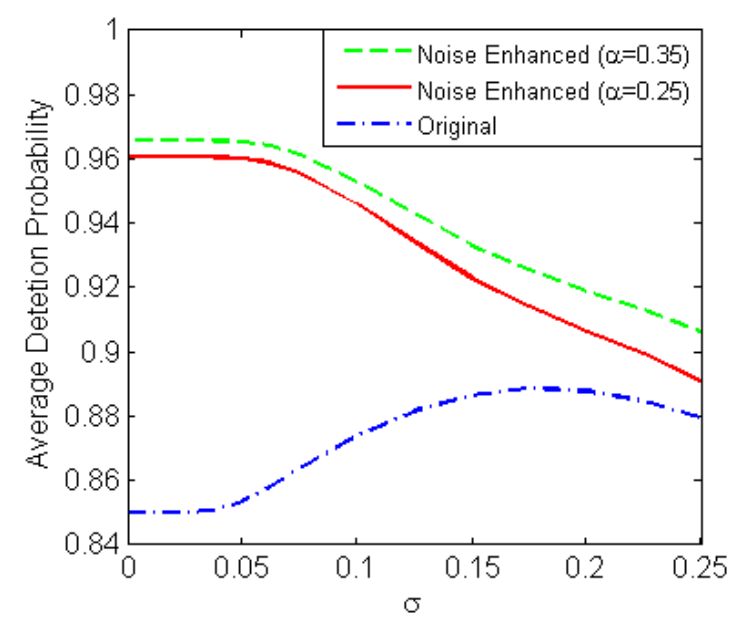

Figure 3. Average detection probabilities versus $\sigma$ obtained by the original detector and the noise-enhanced restricted NP approach for $\alpha=0.35$ and 0.25 when $A=1, \tau=0.7$, and $\beta=0.8$.

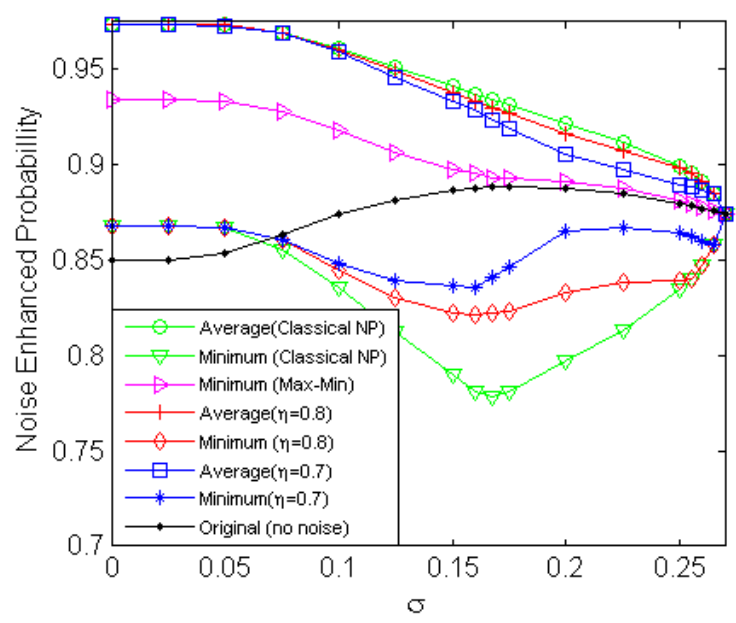

Figure 4. Average and minimum detection probabilities versus $\sigma$ obtained by the original detector and the noise-enhanced restricted NP (for $\eta=0.8$ and 0.7), classical NP, and Max-min approaches when $A=1, \sigma=0.2$, and $\alpha=0.25$.

When $\sigma$ is close to 0 , the average detection probability and the corresponding minimum detection probability obtained by the noise-enhanced restricted NP approach are the same as those obtained by the noise-enhanced classical NP approach, and the minimum detection probability is still greater than the original detection probability. In addition, the change of $\eta$ has little or no effect on the optimal additive noise in this case. With the increase of $\sigma$, the average detection probabilities obtained in the three noise-enhanced approaches decrease gradually; the corresponding minimum probabilities first decrease and then increase slightly. The minimum detection probability obtained by the noise-enhanced Max-min approach decreases and gradually approaches the original detection probability. Furthermore, for a certain range of $\sigma$, a greater average detection probability and a smaller minimum detection probability are obtained by the noise-enhanced restricted NP approach for a greater value of $\eta$. In addition, no improvement of the detectability can be achieved by adding any noise when $\sigma$ exceeds a certain level. 


\section{Conclusions}

In this paper, the noise-enhanced signal detection for the composite hypothesis problem is studied according to various criteria. The noise-enhanced detection problem is formulated as a noise-enhanced classical Neyman-Pearson (NP), Max-min, or restricted NP problem when the prior information is completely known, completely unknown, or partially known, respectively. The relationships of the noise-enhanced restricted NP problem, classical NP problem, and Max-min problem are discussed. Further, the optimal additive noise obtained according to the restricted NP criterion was analyzed from a special perspective where the noise-enhanced restricted NP problem is equivalent to a noise-enhanced classical NP problem with different prior distributions under certain conditions, and the related algorithm is provided to find the optimal solution. The minimum detection probability corresponding to the maximum noise-modified average detection probability is proven to equal $\beta$, which is the lower limit of detection probability in the restricted NP framework. In addition, it is demonstrated that the maximum noise-modified average detection probability obtained by the restricted NP approach is a strictly decreasing and concave function of $\beta$. Finally, numerical examples and simulation results are provided to illustrate and verify the theoretical analysis.

In conclusion, the detection performance can be improved by adding additive noise obtained by the restricted NP, classical NP, and Max-min approaches. Specifically, a better Receiver Operating Characteristic (ROC) can be obtained. Under certain conditions, a better ROC means an increase of SNR. Therefore, we could consider extending the theoretical results in this paper to increase the SNR in the quantum regime, or to explore the optimal additive noise that enhances the quantum resonance [3-7]. For example, a superconducting quantum interference device (SQUID) is usually used to convert an applied magnetic flux into a voltage signal [5-7]. First, we may treat the applied magnetic flux as the system input, and then obtain different output voltages via the addition of different direct current (DC) signals to the input. Thus, the corresponding noise-modified SNRs could be calculated. If there is no constraint on the input and/or output, the optimal additive noise would be the DC signal corresponding to the maximum noise-modified SNR.

Acknowledgments: This research is partly supported by the Basic and Advanced Research Project in Chongqing (Grant No. cstc2016jcyjA0134, No. cstc2016jcyjA0043) and the National Natural Science Foundation of China (Grant No. 61501072, No. 61471073, No. 41404027, and No. 61301224).

Author Contributions: Shujun Liu raised the idea of the new framework to solve different noise-enhanced composite hypothesis-testing problems under different criteria. Ting Yang and Shujun Liu contributed to the drafting of the manuscript, interpretation of the results, some experimental design, and checked the manuscript. Mingchun Tang and Hongqing Liu designed the experiment of maximum noise-modified average detection probabilities obtained by the restricted NP approach. Kui Zhang and Xinzheng Zhang contributed to the proofs of the theories developed in this paper. All authors have read and approved the final manuscript.

Conflicts of Interest: The authors declare no conflict of interest.

\section{Appendix A}

Theorem A1. Define a PDF of $\theta$ under $H_{1}$ as $r_{1}(\theta)=\eta \omega_{1}(\theta)+(1-\eta) g(\theta)$, where $g(\theta)$ is any valid PDF. If there exists a PDF $p_{\mathbf{n}}^{*}(\mathbf{n})$ such that

$$
\int_{\mathbb{R}^{N}} p_{\mathbf{n}}^{*}(\mathbf{n}) \int_{\Lambda_{1}} f_{\theta}(\mathbf{n}) g(\theta) d \theta d \mathbf{n}=\min _{\theta \in \Lambda_{1}} \int_{\mathbb{R}^{N}} p_{\mathbf{n}}^{*}(\mathbf{n}) f_{\theta}(\mathbf{n}) d \mathbf{n},
$$

where $p_{\mathbf{n}}^{*}(\mathbf{n})$ is the optimal additive noise PDF to maximize the average detection probability based on $r_{1}(\theta)$ under the constraint that $\max _{\theta \in \Lambda_{1}} \int_{\mathbb{R}^{N}} p_{\mathbf{n}}(\mathbf{n}) f_{\theta}(\mathbf{n}) d \mathbf{n} \leq \alpha$, then $p_{\mathbf{n}}^{*}(\mathbf{n})$ is the optimal solution of Equations (18) and (19). 
Proof.

$$
\begin{aligned}
& \eta \int_{\mathbb{R}^{N}} p_{\mathbf{n}}(\mathbf{n}) f_{1}(\mathbf{n}) d \mathbf{n}+(1-\eta) \min _{\theta \in \Lambda_{1}} \int_{\mathbb{R}^{N}} p_{\mathbf{n}}(\mathbf{n}) f_{\theta}(\mathbf{n}) d \mathbf{n} \\
& \leq \eta \int_{\mathbb{R}^{N}} p_{\mathbf{n}}(\mathbf{n}) \int_{\Lambda_{1}} f_{\theta}(\mathbf{n}) \omega_{1}(\theta) d \theta d \mathbf{n}+(1-\eta) \int_{\Lambda_{1}} \int_{\mathbb{R}^{N}} p_{\mathbf{n}}(\mathbf{n}) f_{\theta}(\mathbf{n}) d \mathbf{n} g(\theta) d \theta \\
& =\eta \int_{\mathbb{R}^{N}} p_{\mathbf{n}}(\mathbf{n}) \int_{\Lambda_{1}} f_{\theta}(\mathbf{n}) \omega_{1}(\theta) d \theta d \mathbf{n}+(1-\eta) \int_{\mathbb{R}^{N}} p_{\mathbf{n}}(\mathbf{n}) \int_{\Lambda_{1}} f_{\theta}(\mathbf{n}) g(\theta) d \theta d \mathbf{n} \\
& =\int_{\mathbb{R}^{N}} p_{\mathbf{n}}(\mathbf{n}) \int_{\Lambda_{1}} f_{\theta}(\mathbf{n})\left(\eta \omega_{1}(\theta)+(1-\eta) g(\theta) d \theta d \mathbf{n}\right. \\
& =\int_{\mathbb{R}^{N}} p_{\mathbf{n}}(\mathbf{n}) \int_{\Lambda_{1}} f_{\theta}(\mathbf{n}) r_{1}(\theta) d \theta d \mathbf{n}
\end{aligned}
$$

where the first inequality holds because $\min _{\theta \in \Lambda_{1}} \int_{\mathbb{R}^{N}} p_{\mathbf{n}}(\mathbf{n}) f_{\theta}(\mathbf{n}) d \mathbf{n} \leq \int_{\Lambda_{1}} \int_{\mathbb{R}^{N}} p_{\mathbf{n}}(\mathbf{n}) f_{\theta}(\mathbf{n}) d \mathbf{n} g(\theta) d \theta$, and the equality holds if and only if $\min _{\theta \in \Lambda_{1}} \int_{\mathbb{R}^{N}} p_{\mathbf{n}}(\mathbf{n}) f_{\theta}(\mathbf{n}) d \mathbf{n}=\int_{\Lambda_{1}} \int_{\mathbb{R}^{N}} p_{\mathbf{n}}(\mathbf{n}) f_{\theta}(\mathbf{n}) d \mathbf{n} g(\theta) d \theta$.

Since $p_{\mathbf{n}}^{*}(\mathbf{n})$ is the optimal additive noise PDF corresponding to the maximum average detection probability based on $r_{1}(\theta)$ and under the constraint that $\max _{\theta \in \Lambda_{1}} \int_{\mathbb{R}^{N}} p_{\mathbf{n}}(\mathbf{n}) f_{\theta}(\mathbf{n}) d \mathbf{n} \leq \alpha$, we have

$$
\int_{\mathbb{R}^{N}} p_{\mathbf{n}}(\mathbf{n}) \int_{\Lambda_{1}} f_{\theta}(\mathbf{n}) r_{1}(\theta) d \theta d \mathbf{n} \leq \int_{\mathbb{R}^{N}} p_{\mathbf{n}}^{*}(\mathbf{n}) \int_{\Lambda_{1}} f_{\theta}(\mathbf{n}) r_{1}(\theta) d \theta d \mathbf{n}
$$

If $\int_{\mathbb{R}^{N}} p_{\mathbf{n}}^{*}(\mathbf{n}) \int_{\Lambda_{1}} f_{\theta}(\mathbf{n}) g(\theta) d \theta d \mathbf{n}=\min _{\theta \in \Lambda_{1}} \int_{\mathbb{R}^{N}} p_{\mathbf{n}}^{*}(\mathbf{n}) f_{\theta}(\mathbf{n}) d \mathbf{n}$, we have

$$
\begin{aligned}
& \eta \int_{\mathbb{R}^{N}} p_{\mathbf{n}}^{*}(\mathbf{n}) \int_{\Lambda_{1}} f_{\theta}(\mathbf{n}) \omega_{1}(\theta) d \theta d \mathbf{n}+(1-\eta) \int_{\Lambda_{1}} \int_{\mathbb{R}^{N}} p_{\mathbf{n}}^{*}(\mathbf{n}) f_{\theta}(\mathbf{n}) d \mathbf{n} g(\theta) d \theta \\
& =\eta \int_{\mathbb{R}^{N}} p_{\mathbf{n}}^{*}(\mathbf{n}) f_{1}(\mathbf{n}) d \mathbf{n}+(1-\eta) \min _{\theta \in \Lambda_{1}} \int_{\mathbb{R}^{N}} p_{\mathbf{n}}^{*}(\mathbf{n}) f_{\theta}(\mathbf{n}) d \mathbf{n}
\end{aligned}
$$

In conclusion, $p_{\mathbf{n}}^{*}(\mathbf{n})$ is the solution of Equations (18) and (19). In addition, Equation (18) is always less than or equal to (A4).

Corollary A1. Under the conditions in Theorem 1, if $\min _{\theta \in \Lambda_{1}} \int_{\mathbb{R}^{N}} p_{\mathbf{n}}^{*}(\mathbf{n}) f_{\theta}(\mathbf{n}) d \mathbf{n}=\beta, p_{\mathbf{n}}^{*}(\mathbf{n})$ is the optimal additive noise PDF corresponding to the optimization problem described in Equations (10) and (11).

Proof. From the definition of $p_{\mathbf{n}}^{*}(\mathbf{n})$ in Theorem $1, p_{\mathbf{n}}^{*}(\mathbf{n})$ achieves the maximum of Equation (18), and the following inequality always holds under the constraint that $\max _{\theta \in \Lambda_{1}} \int_{\mathbb{R}^{N}} p_{\mathbf{n}}(\mathbf{n}) f_{\theta}(\mathbf{n}) d \mathbf{n} \leq \alpha$. That is

$$
\begin{aligned}
& \eta \int_{\mathbb{R}^{N}} p_{\mathbf{n}}(\mathbf{n}) f_{1}(\mathbf{n}) d \mathbf{n}+(1-\eta) \min _{\theta \in \Lambda_{1}} \int_{\mathbb{R}^{N}} p_{\mathbf{n}}(\mathbf{n}) f_{\theta}(\mathbf{n}) d \mathbf{n} \\
\leq & \eta \int_{\mathbb{R}^{N}} p_{\mathbf{n}}^{*}(\mathbf{n}) f_{1}(\mathbf{n}) d \mathbf{n}+(1-\eta) \min _{\theta \in \Lambda_{1}} \int_{\mathbb{R}^{N}} p_{\mathbf{n}}^{*}(\mathbf{n}) f_{\theta}(\mathbf{n}) d \mathbf{n} .
\end{aligned}
$$

Since $\min _{\theta \in \Lambda_{1}} \int_{\mathbb{R}^{N}} p_{\mathbf{n}}(\mathbf{n}) f_{\theta}(\mathbf{n}) d \mathbf{n} \geq \beta$ according to Equation (11) and $\min _{\theta \in \Lambda_{1}} \int_{\mathbb{R}^{N}} p_{\mathbf{n}}^{*}(\mathbf{n}) f_{\theta}(\mathbf{n}) d \mathbf{n}=\beta$ as assumed in the corollary, $\int_{\mathbb{R}^{N}} p_{\mathbf{n}}(\mathbf{n}) f_{1}(\mathbf{n}) d \mathbf{n}$ should be less than or equal to $\int_{\mathbb{R}^{N}} p_{\mathbf{n}}^{*}(\mathbf{n}) f_{1}(\mathbf{n}) d \mathbf{n}$ in order to make the inequality in (A5) true. Therefore, when $\min _{\theta \in \Lambda_{1}} \int_{\mathbb{R}^{N}} p_{\mathbf{n}}^{*}(\mathbf{n}) f_{\theta}(\mathbf{n}) d \mathbf{n}=\beta$ under the conditions in Theorem 1, $p_{\mathbf{n}}^{*}(\mathbf{n})$ is the solution of Equations (10) and (11).

Theorem A2. Under the conditions in Theorem $1, r_{1}(\theta)=\eta \omega_{1}(\theta)+(1-\eta) g(\theta)$ is the PDF that minimizes the noise-modified average detection probability among all probability distributions of the form $\hat{r}_{1}(\theta)=\hat{\eta} \omega_{1}(\theta)+$ $(1-\hat{\eta}) \hat{g}(\theta)$, where $\hat{\eta} \geq \eta, \theta \in \Lambda_{1}$ and $\hat{g}(\theta)$ is any PDF. In other words, the following inequality holds:

$$
\int_{\mathbb{R}^{N}} p_{\mathbf{n}}^{*}(\mathbf{n}) \int_{\Lambda_{1}} f_{\theta}(\mathbf{n}) r_{1}(\theta) d \theta d \mathbf{n} \leq \int_{\mathbb{R}^{N}} \hat{p}_{\mathbf{n}}^{*}(\mathbf{n}) \int_{\Lambda_{1}} f_{\theta}(\mathbf{n}) \hat{r}_{1}(\theta) d \theta d \mathbf{n},
$$

where $p_{\mathbf{n}}^{*}(\mathbf{n})$ and $\hat{p}_{\mathbf{n}}^{*}(\mathbf{n})$ are the optimal additive noise PDFs obtained by the classical NP approach corresponding to $r_{1}(\theta)$ and $\hat{r}_{1}(\theta)$, respectively. 
Proof. Under the conditions in Theorem 1, we have

$$
\begin{aligned}
& \int_{\mathbb{R}^{N}} p_{\mathbf{n}}^{*}(\mathbf{n}) \int_{\Lambda_{1}} f_{\theta}(\mathbf{n}) r_{1}(\theta) d \theta d \mathbf{n} \\
& =\eta \int_{\mathbb{R}^{N}} p_{\mathbf{n}}^{*}(\mathbf{n}) \int_{\Lambda_{1}} f_{\theta}(\mathbf{n}) \omega_{1}(\theta) d \theta d \mathbf{n}+(1-\eta) \int_{\Lambda_{1}} \int_{\mathbb{R}^{N}} p_{\mathbf{n}}^{*}(\mathbf{n}) f_{\theta}(\mathbf{n}) d \mathbf{n} g(\theta) d \theta . \\
& =\eta \int_{\mathbb{R}^{N}} p_{\mathbf{n}}^{*}(\mathbf{n}) \int_{\Lambda_{1}} f_{\theta}(\mathbf{n}) \omega_{1}(\theta) d \theta d \mathbf{n}+(1-\eta) \min _{\theta \in \Lambda_{1}} \int_{\mathbb{R}^{N}} p_{\mathbf{n}}^{*}(\mathbf{n}) f_{\theta}(\mathbf{n}) d \mathbf{n}
\end{aligned}
$$

Since $\int_{\mathbb{R}^{N}} p_{\mathbf{n}}^{*}(\mathbf{n}) \int_{\Lambda_{1}} f_{\theta}(\mathbf{n}) \omega_{1}(\theta) d \theta d \mathbf{n} \geq \min _{\theta \in \Lambda_{1}} \int_{\mathbb{R}^{N}} p_{\mathbf{n}}^{*}(\mathbf{n}) f_{\theta}(\mathbf{n}) d \mathbf{n}$, for any $\hat{\eta} \geq \eta$, one obtains

$$
\begin{aligned}
& \int_{\mathbb{R}^{N}} p_{\mathbf{n}}^{*}(\mathbf{n}) \int_{\Lambda_{1}} f_{\theta}(\mathbf{n}) r_{1}(\theta) d \theta d \mathbf{n} \\
& \leq \hat{\eta} \int_{\mathbb{R}^{N}} p_{\mathbf{n}}^{*}(\mathbf{n}) \int_{\Lambda_{1}} f_{\theta}(\mathbf{n}) \omega_{1}(\theta) d \theta d \mathbf{n}+(1-\hat{\eta}) \min _{\theta \in \Lambda_{1}} \int_{\mathbb{R}^{N}} p_{\mathbf{n}}^{*}(\mathbf{n}) f_{\theta}(\mathbf{n}) d \mathbf{n} \\
& \leq \hat{\eta} \int_{\mathbb{R}^{N}} p_{\mathbf{n}}^{*}(\mathbf{n}) \int_{\Lambda_{1}} f_{\theta}(\mathbf{n}) \omega_{1}(\theta) d \theta d \mathbf{n}+(1-\hat{\eta}) \int_{\Lambda_{1}} \hat{g}(\theta) \int_{\mathbb{R}^{N}} p_{\mathbf{n}}^{*}(\mathbf{n}) f_{\theta}(\mathbf{n}) d \mathbf{n} d \theta . \\
& =\int_{\mathbb{R}^{N}} p_{\mathbf{n}}^{*}(\mathbf{n}) \int_{\Lambda_{1}} f_{\theta}(\mathbf{n})\left(\hat{\eta} \omega_{1}(\theta)+(1-\hat{\eta}) \hat{g}(\theta)\right) d \theta d \mathbf{n} \\
& =\int_{\mathbb{R}^{N}} p_{\mathbf{n}}^{*}(\mathbf{n}) \int_{\Lambda_{1}} f_{\theta}(\mathbf{n}) \hat{r}_{1}(\theta) d \theta d \mathbf{n} \\
& \leq \int_{\mathbb{R}^{N}} \hat{p}_{\mathbf{n}}^{*}(\mathbf{n}) \int_{\Lambda_{1}} f_{\theta}(\mathbf{n}) \hat{r}_{1}(\theta) d \theta d \mathbf{n}
\end{aligned}
$$

The last inequality holds due to the definition of $\hat{p}_{\mathbf{n}}^{*}(\mathbf{n})$. From Equation (A8), the maximum noise-modified average detection probability corresponding to $r_{1}(\theta)=\eta \omega_{1}(\theta)+(1-\eta) g(\theta)$ is less than or equal to that corresponding to $\hat{r}_{1}(\theta)=\hat{\eta} \omega_{1}(\theta)+(1-\hat{\eta}) \hat{g}(\theta)$ for $\hat{\eta} \geq \eta$.

Theorem A3. When $\beta \in[C, M]$, the maximum noise-modified detection probability $P_{D}^{a v g}\left(p_{\mathbf{n}}^{\beta}(\mathbf{n})\right)$ obtained by the restricted NP approach is a strictly decreasing and concave function of $\beta$.

Proof. First, based on the definition of the noise-enhanced restricted NP approach, $P_{D}^{a v g}\left(p_{\mathbf{n}}^{\beta}(\mathbf{n})\right)$ is a non-increasing function of $\beta$. In order to prove the concavity of $P_{D}^{a v g}\left(p_{\mathbf{n}}^{\beta}(\mathbf{n})\right)$ with respect to (w.r.t.) $\beta$, we define an additive noise with PDF $p_{\mathbf{n}}(\mathbf{n})$, which is a convex combination of two optimal additive noises obtained by the restricted NP approach under the same constraint on false-alarm probability corresponding to $\beta=\beta_{1}$ and $\beta=\beta_{2}$. That is,

$$
p_{\mathbf{n}}(\mathbf{n})=\lambda p_{\mathbf{n}}^{\beta_{1}}(\mathbf{n})+(1-\lambda) p_{\mathbf{n}}^{\beta_{2}}(\mathbf{n}),
$$

where $C \leq \beta_{1}<\beta_{2} \leq M, 0<\lambda<1, p_{\mathbf{n}}^{\beta_{1}}(\mathbf{n})$, and $p_{\mathbf{n}}^{\beta_{2}}(\mathbf{n})$ denote the optimal additive noise PDFs obtained by the restricted NP approach for $\beta=\beta_{1}$ and $\beta=\beta_{2}$, respectively. From the definition of $p_{\mathbf{n}}(\mathbf{n})$, the noise-modified detection and false-alarm probabilities corresponding to $p_{\mathbf{n}}(\mathbf{n})$ for a given value of $\theta$ can be obtained as

$$
\begin{gathered}
P_{D}\left(p_{\mathbf{n}}(\mathbf{n}), \theta\right)=\lambda P_{D}\left(p_{\mathbf{n}}^{\beta_{1}}(\mathbf{n}), \theta\right)+(1-\lambda) P_{D}\left(p_{\mathbf{n}}^{\beta_{2}}(\mathbf{n}), \theta\right), \theta \in \Lambda_{1}, \\
P_{F A}\left(p_{\mathbf{n}}(\mathbf{n}), \theta\right)=\lambda P_{F A}\left(p_{\mathbf{n}}^{\beta_{1}}(\mathbf{n}), \theta\right)+(1-\lambda) P_{F A}\left(p_{\mathbf{n}}^{\beta_{2}}(\mathbf{n}), \theta\right), \theta \in \Lambda_{0} .
\end{gathered}
$$

From the relation in Equation (A11), it is obvious that $p_{\mathbf{n}}(\mathbf{n})$ also satisfies the constraint on false-alarm probability. That is,

$$
\max _{\theta \in \Lambda_{0}} P_{F A}\left(p_{\mathbf{n}}(\mathbf{n}), \theta\right) \leq \lambda \max _{\theta \in \Lambda_{0}} P_{F A}\left(p_{\mathbf{n}}^{\beta_{1}}(\mathbf{n}), \theta\right)+(1-\lambda) \max _{\theta \in \Lambda_{0}} P_{F A}\left(p_{\mathbf{n}}^{\beta_{2}}(\mathbf{n}), \theta\right) \leq \alpha .
$$

Based on Equations (A9) and (A10), the noise-modified average detection probability corresponding to $p_{\mathbf{n}}(\mathbf{n})$ can be calculated by

$$
P_{D}^{a v g}\left(p_{\mathbf{n}}(\mathbf{n})\right)=\lambda P_{D}^{a v g}\left(p_{\mathbf{n}}^{\beta_{1}}(\mathbf{n})\right)+(1-\lambda) P_{D}^{a v g}\left(p_{\mathbf{n}}^{\beta_{2}}(\mathbf{n})\right) .
$$


Accordingly, the minimum detection probability obtained by adding the noise with $\operatorname{PDF} p_{\mathbf{n}}(\mathbf{n})$ can be upper bounded by

$$
\begin{aligned}
\min _{\theta \in \Lambda_{1}} P_{D}\left(p_{\mathbf{n}}(\mathbf{n}), \theta\right) & \geq \lambda \min _{\theta \in \Lambda_{1}} P_{D}\left(p_{\mathbf{n}}^{\beta_{1}}(\mathbf{n}), \theta\right)+(1-\lambda) \min _{\theta \in \Lambda_{1}} P_{D}\left(p_{\mathbf{n}}^{\beta_{2}}(\mathbf{n}), \theta\right) . \\
& \geq \lambda \beta_{1}+(1-\lambda) \beta_{2}
\end{aligned} .
$$

Let $\min _{\theta \in \Lambda_{1}} P_{D}\left(p_{\mathbf{n}}(\mathbf{n}), \theta\right)=\beta$ and $\hat{\beta}=\lambda \beta_{1}+(1-\lambda) \beta_{2}$, the following inequalities can be obtained according to Equations (A13) and (A14),

$$
P_{D}^{a v g}\left(p_{\mathbf{n}}^{\hat{\beta}}(\mathbf{n})\right) \geq P_{D}^{a v g}\left(p_{\mathbf{n}}^{\beta}(\mathbf{n})\right) \geq P_{D}^{a v g}\left(p_{\mathbf{n}}(\mathbf{n})\right)=\lambda P_{D}^{a v g}\left(p_{\mathbf{n}}^{\beta_{1}}(\mathbf{n})\right)+(1-\lambda) P_{D}^{a v g}\left(p_{\mathbf{n}}^{\beta_{2}}(\mathbf{n})\right)
$$

where the first inequality holds due to the non-increasing character of $P_{D}^{a v g}\left(p_{\mathbf{n}}^{\beta}(\mathbf{n})\right)$ w.r.t. $\beta$ and the second inequality follows because $p_{\mathbf{n}}^{\beta}(\mathbf{n})$ is the optimal additive noise to maximize the noise-modified average detection probability for the case where the constraint on the minimum detection probability is selected as $\beta$. Therefore, $P_{D}^{a v g}\left(p_{\mathbf{n}}^{\beta}(\mathbf{n})\right)$ is proven to be a concave function w.r.t. $\beta$.

Next, the strictly decreasing character of $P_{D}^{a v g}\left(p_{\mathbf{n}}^{\beta}(\mathbf{n})\right)$ will be shown. Let $C<\beta_{1}<\beta_{2} \leq M$ and suppose $P_{D}^{a v g}\left(p_{\mathbf{n}}^{\beta_{1}}(\mathbf{n})\right)=P_{D}^{a v g}\left(p_{\mathbf{n}}^{\beta_{2}}(\mathbf{n})\right)$, which means $p_{\mathbf{n}}^{\beta_{2}}(\mathbf{n})$ is also an optimal additive noise corresponding to $\beta_{1}$. We then have $\min _{\theta \in \Lambda_{1}} P_{D}\left(p_{\mathbf{n}}^{\beta_{1}}(\mathbf{n}), \theta\right) \geq \beta_{2}>\beta_{1}$, which obviously contradicts Equation (31). Therefore, $P_{D}^{a v g}\left(p_{\mathbf{n}}^{\beta_{1}}(\mathbf{n})\right)<P_{D}^{a v g}\left(p_{\mathbf{n}}^{\beta_{2}}(\mathbf{n})\right)$ must satisfy for any $C<\beta_{1}<\beta_{2} \leq M$.

In summary, $P_{D}^{a v g}\left(p_{\mathbf{n}}^{\beta}(\mathbf{n})\right)$ is a strictly decreasing concave function w.r.t. $\beta$.

\section{References}

1. Benzi, R.; Sutera, A.; Vulpiani, A. The mechanism of stochastic resonance. J. Phys. A Math. Gen. 1981, 14, 453-457. [CrossRef]

2. Löfstedt, R.; Coppersmith, S.N. Quantum stochastic resonance. Phys. Rev. Lett. 1994, 72, 1947-1950. [CrossRef] [PubMed]

3. Grifoni, M.; Hänggi, P. Nonlinear quantum stochastic resonance. Phys. Rev. E 1996, 54, 1390-1401. [CrossRef]

4. Hibbs, A.D.; Singsaas, A.L. Stochastic resonance in a superconducting loop with a Josephson junction. J. Appl. Phys. 1995, 77, 2582-2590. [CrossRef]

5. Rouse, R.; Han, S.; Lukens, J.E. Flux amplification using stochastic superconducting quantum interference devices. Appl. Phys. Lett. 1995, 66, 108-110. [CrossRef]

6. Glukhov, A.M.; Sivakov, A.G. Observation of stochastic resonance in percolative Josephson media. Low Temp. Phys. 2002, 28, 383-386. [CrossRef]

7. Patel, A.; Kosko, B. Stochastic resonance in continuous and spiking neuron models with Levy noise. IEEE Trans. Neural Netw. 2008, 19, 1993-2008. [CrossRef] [PubMed]

8. Addesso, P.; Filatrella, G.; Pierro, V. Characterization of escape times of Josephson junctions for signal detection. Phys. Rev. E 2012, 85, 016708. [CrossRef] [PubMed]

9. Weber, J.F.; Waldman, S.D. Stochastic Resonance is a Method to Improve the Biosynthetic Response of Chondrocytes to Mechanical Stimulation. J. Orthop. Res. 2015, 34, 231-239. [CrossRef] [PubMed]

10. Zozor, S.; Ambland, P.O. On the use of stochastic resonance in sine detection. IEEE Process. 2002, 7, 353-367. [CrossRef]

11. Zozor, S.; Ambland, P.O. Stochastic resonance in locally optimal detectors. IEEE Process. 2002, 51, 3177-3181. [CrossRef]

12. Patel, A.; Kosko, B. Noise benefits in quantizer-array correlation detection and watermark decoding. IEEE Trans. Signal Process. 2011, 59, 488-505. [CrossRef]

13. Chen, H.; Varshney, L.R.; Varshney, P.K. Noise-enhanced information systems. Proc. IEEE 2014, 102, 1607-1621. [CrossRef] 
14. Han, D.; Li, P.; An, S.; Shi, P. Multi-frequency weak signal detection based on wavelet transform and parameter compensation band-pass multi-stable stochastic resonance. Mech. Syst. Signal Process. 2016, 70, 995-1010. [CrossRef]

15. Addesso, P.; Pierro, V.; Filatrella, G. Interplay between detection strategies and stochastic resonance properties. Commun. Nonlinear Sci. Numer. Simul. 2016, 30, 15-31. [CrossRef]

16. Stocks, N.G. Suprathreshold stochastic resonance in multilevel threshold systems. Phys. Rev. Lett. 2000, 84, 2310-2313. [CrossRef] [PubMed]

17. Kosko, B.; Mitaim, S. Stochastic resonance in noisy threshold neurons. Neural Netw. 2003, 16, 755-761. [CrossRef]

18. Kosko, B.; Mitaim, S. Robust stochastic resonance for simple threshold neurons. Phys. Rev. E 2004, 70, 031911. [CrossRef] [PubMed]

19. Mitaim, S.; Kosko, B. Adaptive stochastic resonance in noisy neurons based on mutual information. IEEE Trans. Neural Netw. 2004, 15, 1526-1540. [CrossRef] [PubMed]

20. Gingl, Z.; Makra, P.; Vajtai, R. High signal-to-noise ratio gain by stochastic resonance in a double well. Fluct. Noise Lett. 2001, 1, L181-L188. [CrossRef]

21. Makra, P.; Gingl, Z. Signal-to-noise ratio gain in non-dynamical and dynamical bistable stochastic resonators. Fluct. Noise Lett. 2002, 2, L145-L153. [CrossRef]

22. Makra, P.; Gingl, Z.; Fulei, T. Signal-to-noise ratio gain in stochastic resonators driven by coloured noises. Phys. Lett. A 2003, 317, 228-232. [CrossRef]

23. Kay, S. Can detectability be improved by adding noise? IEEE Signal Process. Lett. 2000, 7, 8-10. [CrossRef]

24. Chen, H.; Varshney, P.K.; Kay, S.M.; Michels, J.H. Theory of the stochastic resonance effect in signal detection: Part I-Fixed detectors. IEEE Trans. Signal Process. 2007, 55, 3172-3184. [CrossRef]

25. Chen, H.; Varshney, P.K.; Kay, S.M.; Michels, J.H. Theory of the stochastic resonance effect in signal detection: Part II-Variable detectors. IEEE Trans. Signal Process. 2007, 56, 5031-5041. [CrossRef]

26. Patel, A.; Kosko, B. Optimal noise benefits in Neyman-Pearson and inequality constrained signal detection. IEEE Trans. Signal Process. 2009, 57, 1655-1669. [CrossRef]

27. Bayram, S.; Gezici, S. On the Restricted Neyman-Pearson Approach for composite hypothesis-testing in presence of prior distribution uncertainty. IEEE Trans. Signal Process. 2011, 59, 5056-5065. [CrossRef]

28. Bayram, S.; Gezici, S. Stochastic resonance in binary composite hypothesis-testing problems in the Neyman-Pearson framework. Digit. Signal Process. 2012, 22, 391-406. [CrossRef]

29. Bayram, S.; Gultekin, S.; Gezici, S. Noise enhanced hypothesis-testing according to restricted Neyman-Pearson criterion. Digit. Signal Process. 2014, 25, 17-27. [CrossRef]

30. Bayram, S.; Gezici, S. Noise-enhanced M-ary hypothesis-testing in the mini-max framework. In Proceedings of the 3rd International Conference on Signal Processing and Communication Systems, Omaha, NE, USA, 28-30 September 2009; pp. 1-6.

31. Bayram, S.; Gezici, S.; Poor, H.V. Noise enhanced hypothesis-testing in the restricted bayesian framework. IEEE Trans. Signal Process. 2010, 58, 3972-3989. [CrossRef]

32. Bayram, S.; Gezici, S. Noise enhanced M-ary composite hypothesis-testing in the presence of partial prior information. IEEE Trans. Signal Process. 2011, 59, 1292-1297. [CrossRef]

33. Kay, S.M.; Michels, J.H.; Chen, H.; Varshney, P.K. Reducing probability of decision error using stochastic resonance. IEEE Signal Process. Lett. 2006, 13, 695-698. [CrossRef]

34. Lehmann, E.L. Testing Statistical Hypotheses, 2nd ed.; Chapman \& Hall: New York, NY, USA, 1986.

35. Richards, M.A. Fundamentals of Radar Signal Processing; McGraw-Hill Profrssional Engineering: New York, NY, USA, 2005.

36. Zarrin, S.; Lim, T.J. Composite hypothesis testing for cooperative spectrum sensing in cognitive radio. In Proceedings of the IEEE International Conference on Communications, Dresden, Germany, 14-18 June 2009; pp. 1721-1725.

37. Hodges, J.L., Jr.; Lehmann, E.L. The use of previous experience in reaching statistical decisions. Ann. Math. Stat. 1952, 23, 396-407. [CrossRef]

(C) 2016 by the authors; licensee MDPI, Basel, Switzerland. This article is an open access article distributed under the terms and conditions of the Creative Commons Attribution (CC-BY) license (http://creativecommons.org/licenses/by/4.0/). 